\title{
Artificial Intuition in Tech Journalism on Al: Imagining the Human Subject
}

\author{
Jacob Johanssen ${ }^{1}$ (D) and Xin Wang ${ }^{2}$ \\ 1 Institute of Business, Law and Society, St. Mary's University, Twickenham, London, UK \\ 2 College of Arts and Media, Tongji University, Shanghai, People's Republic of China
}

\begin{abstract}
Artificial intuition (Al acting intuitively) is one trend in artificial intelligence. This article analyzes how it is discussed by technology journalism on the internet. The journalistic narratives that were analyzed claim that intuition can make Al more efficient, autonomous, and human. Some commentators also write that intuitive Al could execute tasks better than humans themselves ever could (e.g., in digital games); therefore, it could ultimately surpass human intuition. Such views do not pay enough attention to biases as well as transparency and explainability of Al. We contrast the journalistic narratives with philosophical understandings of intuition and a psychoanalytic view of the human. Those perspectives allow for a more complex view that goes beyond the focus on rationality and computational perspectives of tech journalism.
\end{abstract}

Keywords: Artificial Intelligence (Al), artificial intuition, human subject, psychoanalysis, technology journalism

\section{Introduction}

Artificial Intelligence (AI) is an evolving and significant technology. It is increasingly discussed in the media and there are many dedicated popular journalistic websites that cover the latest trends and ideas of AI development (Bory, 2019; Brennen et al., 2018; Goode, 2018; Natale \& Ballatore, 2017). Such discussions are often characterized by "magical thinking,"

CONTACT Dr. Jacob Johanssen (10) - Senior Lecturer in Communications • Institute of Business, Law and Society • St. Mary's University, Twickenham • London, TW1 4SX • United Kingdom • jacob.johanssen@stmarys.ac.uk 
hyped up and exaggerated claims, and not necessarily very balanced (Goode, 2018, p. 186). AI seems capable of inspiring popular accounts that show the authors' amazement, enthusiasm for, but also fear of, the technology (Cave et al., 2018).

Rather than focusing on the accuracy, the authors' emotional investment, or implied audience response to AI media coverage as some scholars have done, this article follows a different aim. In focusing on one specific trend of contemporary AI, not AI as a whole, we are interested in analyzing it in relation to the following research question: What kind of view of the human subject is articulated in technology journalism about artificial intuition? This broad question refers to both how artificial intuition is discussed in relation to alleged human characteristics that are inherent to the technology, and what kind of view of the human in general is evident in the examined articles. We pay specific attention to how this phenomenon is being discussed on journalistic websites with a focus on technology (the websites iTech Post, Toolbox, TechXplore, Inverse, Business World, Science Mag, TechCrunch, Science Daily, Venture Beat, KDnuggets, Hackermoon, Inc, and Hadean were part of the sample) which cater for a particular audience, rather than more "mainstream" quality journalistic websites such as those of broadsheet or tabloid newspapers. Brennen et al. (2020) define technology journalism as coverage of "the technology industry" (p. 16) by which they mean reporting on the products of technology businesses, on technology businesses themselves, and on the social effects of technology. Tech journalism often covers emerging and new technologies, such as artificial intelligence, communication technology, bio- or nanotechnology. Brennen et al. (2020) argue that it is an understudied phenomenon and "there is a rich potential for future studies of technology journalism" (p. 15). Artificial intuition is so recent and somewhat niche that it has not been adequately covered by tabloid or broadsheet journalism yet. This article therefore specifically focuses on online tech journalism. While each sampled website may have its own focus, target audience, style, and mission, all can be seen as belonging to the category of tech journalism. It would be beyond the scope of the initial research into the topic to discuss each sampled outlet in more detail. Journalistic content is sometimes written by individuals who are, for example, data scientists or AI experts rather than journalists. It is also questionable if some of the outlets examined in this article employ the same measures of quality control as traditional journalism as authors for some could self-publish their views on the topic (Brennen et al., 2020). Future research could situate the research findings more in relation to the specificities of the different outlets of tech journalism.

The central point of this article is that the journalistic narratives that were analyzed claim that AI can mimic and adopt characteristics of human intuition. The data we discuss show a view of the human subject that articulates that AI can both learn from and that it can be better and more advanced than human intuition. We argue that such views demonstrate the desire of many journalists who write about AI to make it human-like. It is argued that intuition can make AI more efficient, autonomous, human, and flexible than it currently is. As we discuss in the last section on AI and games, the ultimate hope for some commentators is that tasks can be executed more efficiently than humans themselves ever could. Therefore, intuitive AI could ultimately surpass human intuition. However, such views do not pay enough attention to questions of the human subject as well as transparency, explainability, and accountability of AI. 


\section{Literature Review}

There are many different definitions of AI (see, e.g., Russell \& Norvig, 2010; Turner, 2019 for overviews). Generally, artificial intelligence refers to "the study of agents that receive percepts from the environment and perform actions" (Russell \& Norvig, 2010, p. viii). Such agents are often machines, such as digital computers. Turner defines AI as the "ability of a non-natural entity to make choices by an evaluative process" (p. 16). Non-natural, for Turner, both refers to human-made as well as machine-made (when AI systems create other AI for example).

While AI technologies have been in development since the 1950s, recent years have seen advancements in the complexity of AI when it comes to emulating cognitive characteristics of humans. On a general level, AI has seen increasing developments in the past 10-15 years in particular thanks to the growth of better hardware, data processing, and increased capacity to store and analyze large datasets (Turner, 2019). The seeming ubiquity of AI has led to both an increase in scholarly discussions in the humanities and social sciences as well as AI being picked up by news media. Given that as a technology AI often seeks to simulate or emulate human behavior (such as problem-solving, cognitive abilities, or pattern recognition) and advance such human characteristics, it is unsurprising that it has attracted much attention by journalists and scholars alike.

Artificial intuition refers to the ability of AI systems to make intuitive choices or respond intuitively to problems (Crowder \& Friess, 2013). It was chosen as an example of current discussions of trends in AI that are picked up by journalistic websites with a focus on technology. Questions around intuitive AI have been gaining in prominence in recent years (e.g., when it comes to discussions about self-driving cars or the ability of AI systems that are used for gaming, such as DeepMind's AlphaGo).

Artificial intuition is compared to, for example, neuromorphic hardware, machine learning, or neural networks, more in its infancy (Crowder \& Friess, 2013; Srdanov et al., 2016; Tao \& He, 2009). As we show, there is some hype around artificial intuition. This makes it all the more relevant to analyze. It has also been discussed in literature on AI that comes from a computer science or engineering perspective for some time and has gained more attention in recent years (Caudill \& Butler, 1990; Crowder \& Friess, 2013; DiazHernandez \& Gonzalez-Villela, 2015; Dundas \& Chik, 2011; Frantz, 2003; Johnny et al., 2019; Srdanov et al., 2016; Tao \& He, 2009).

AI acting intuitively may be necessary in certain situations, because decisions can be made quicker than in logical AI. Johnny et al. (2019) define artificial intuition as "the ability of a system to assess a problem context and use pattern recognition or properties from a dataset to choose a course of action or aid the decision process in an automatic manner" (p. 470). Srdanov et al. (2016) propose a trial and error approach which combines logic and randomness in order to reach a specific solution or solve a problem (e.g., making moves in a game). They argue that this provides a way to reach a goal quicker than without trial and error. Diaz-Hernandez and Gonzalez-Villela (2015) suggest that human intuition can be mapped onto artificial intuition through three shared characteristics: inputs, processing, and outputs. In acting intuitively, both users and AI systems unconsciously process inputs, act upon them, and generate outputs or solutions. They tested their model through a robot that picked and placed objects on a surface. Johnny et al. (2019) note that one of the 
potential benefits of artificial intuition is reducing "the complexity of the set of instructions needed to solve the task" (p. 466); for example, in an algorithm.

From a technical perspective, intuition is seen in the above works as "subconscious pattern recognition" (Frantz, 2003, p. 266) of humans and AI systems. All of the authors discussed above seek to translate human intuition into a technical concept which can be operationalized. We argue that the way intuition is approached in those studies is, perhaps paradoxically, anti-intuitive. Intuition is conceptualized as "scientific-rational, logical" (Frantz, 2003, p. 267). This was often mirrored in the way it is presented in the sample of tech journalism articles.

Such forms of rationalistic accounts of intuition are in contrast to how it is theorized in cultural studies and the humanities more generally. In trying to map intuition onto computer science, and thereby essentially making it code-based, the above accounts lose the complexity of intuition and how it relates to dimensions beyond language and consciousness. For instance, human intuition is not the same as a trial and error approach in computation, as Srdanov et al. (2016) claim. While some computer scientists have acknowledged that artificial intuition will remain limited compared to its human counterpart (DiazHernandez \& Gonzalez-Villela, 2015), there is scope to put such scholarship in dialogue with other disciplines.

The term "intuition" has been discussed from many different perspectives. For the purpose of this article we shall largely draw on how intuition has been defined in cultural studies, affect theories, and psychoanalysis (for extensive discussion of the term, see Chudnoff, 2013). Intuition is dependent on an individual human subject and therefore differs for each person. It is commonly understood as a form of knowing that is experienced and felt rather than fully consciously known. It is often associated with terms like gut feeling, hunch, or having a sense of something. Intuition designates a bodily state or experience upon which the individual acts without necessarily having the (empirical) evidence or proof. It is something transitory that is followed or listened to and may then be acted upon without fully understanding why. It refers to a mode of experience within immediacy. It is happening in the now, before being fully rendered conscious and cognitively apprehended by the individual. Intuition is not opposed to, or in contradiction to reason, however. Greg Seigworth (2006) has written that "experience and experiment through the faculty of intuition [...] exceeds or overflows the intellect" (p. 118). For Carolyn Pedwell (2019), intuition "is embodied experience prior to, or in excess of, its translation into the parsing categories of representational and analytical thought" (p. 127). Lauren Berlant (2008) has characterized intuition as an acute source of knowledge. It is a form of "affective intelligence" (p. 852) that shows itself in a particular relational sensitivity to the worldly surroundings of humans.

"With intuition, experience is less a discrete place in the time past belonging to a subject, and more an immanent process of relation (beyond inside and outside, beyond subject and object)" (Seigworth, 2006, p. 119). Such conceptualizations of intuition broaden its theoretical frame to include a relational and open perspective.

We were specifically interested in how human subjectivity is discussed in tech journalism's articles on artificial intuition. This article is informed by a psychoanalytic theory of the human subject. This means that the human subject and specifically their cognitive functions go beyond rationality and mechanistic accounts which often link computational facilities to the brain (Turkle, 2005). According to psychoanalysis, humans are shaped 
by emotional, affective, intuitive, as well as rational actions and feelings (Rosenblatt \& Thickstun, 1994).

Psychoanalysis holds that humans are intuitive, complex, inefficient, contradictory, and sometimes flawed beings. Human beings are influenced by unconscious processes which shape their fantasies, desires, and actions. This emphasis of the unconscious includes considerations of intuition (Board, 1958; Rosenblatt \& Thickstun, 1994; Szalita-Pemow, 1955). Humans regularly make unconscious decisions and this includes intuition but also goes beyond it. For Sigmund Freud, it is both the intuition of the psychoanalyst as well as that of the patient that enables the free associative form of talking in the consulting room during a therapy session (Freud, 1981). For psychoanalysis, then, intuition refers to ways of knowing and experiencing the world where parts of knowledge "are unconscious or otherwise unavailable for conscious delineation” (Board, 1958, p. 237).

\section{Artificial Intelligence in the Media}

The question how artificial intelligence is discussed in the media and in particular what kind of image of the human, is shown through such narratives and has been researched only by a few scholars (Bory, 2019; Brennen et al., 2018; Goode, 2018; Natale \& Ballatore, 2017). Natale and Ballatore (2017) examined early media coverage of AI in technological magazines from the 1950 s to the 1970s. They found that the articles they examined created a "myth" (p. 2) of AI inevitably becoming a thinking machine which would be able to simulate the human brain. Bory compared the coverage on IBM's Deep Blue in 1997 and DeepMind's AlphaGo in 2016. He argues that there has been a shift in narratives from suspicion and conflicted views about Deep Blue in the late 1990s toward recent discourses about AlphaGo that frame it as embodying human characteristics such as beauty or trust while simultaneously being distinctly unhuman-like. This leads to a view of AI as complementary to humans and opening up ways of collaboration rather than competition between the two. Bourne (2019) similarly discusses how AI is framed by PR strategists to promote particular understandings of AI as "friendly" and "competitive." This masks problematic aspects of AI such as its deployment for purposes of discrimination, or harassment. Goode (2018) analyzed mainstream science fiction, media events (such as AlphaGo), and futurology discourses around AI and argues that they are often sensationalist and misleading, for example, when it comes to discourses about a coming artificial mind. Yet, such discourses can also serve as useful entry points to initiate more nuanced public debate about AI, as Goode argues.

Brennen et al. (2018) have analyzed how UK media have covered artificial intelligence over 8 months. They show that there can be a bias toward industry sources in media coverage and that sources from academia, government, and wider civil society were in the minority in their sample. AI is often being politicized in media coverage when it comes to ethical questions such as bias, automation, national security, discrimination, and other issues. Such questions are downplayed by the industry in order not to damage its image. They conclude that there is a risk of covering AI in a one-sided manner. Cave et al. (2018) have analyzed how researchers, communicators, policymakers, and different publics talk about AI. Their analyses show that "[p]revalent AI narratives share dominant characteristics: a focus on embodiment; a tendency towards utopian or dystopian extremes; and a lack of diversity in creators, protagonists, and types of AI" (p. 4). 
No research has specifically focused on tech journalism's coverage of artificial intuition in relation to human subjectivity. There is, thus, scope to further research on the discussion of particular aspects of AI by technology journalism outlets, particularly in relation to how the human subject is imagined in them.

\section{Methodology}

The research that forms the basis of this article specifically focused on one technical aspect of AI and how it was covered by tech journalism websites. The articles were obtained by performing a keyword search using Google UK (general search) with the keywords "artificial intuition" and "artificial AND intuition" as well as "artificial intui ${ }^{*}$ " (to include variations of the word, such as "intuitive") in May 2019 and September 2020. It needs to be acknowledged that using Google as a sampling procedure can be seen as problematic but is nonetheless used by scholars as a form of data collection (Ballatore, 2015; Wouters \& Gerbec, 2006). The researchers' institutions had no access to databases such as Factiva that would specifically include online sources (rather than only newspaper articles). Given the exploratory nature of this study, a broad approach to sampling was taken in order to include a diverse range of websites. Using a newspaper database such as LexisNexis would have restricted the sample, because tech journalism websites are not indexed. Additionally, a keyword search via LexisNexis did not return any results for "artificial intuition" from 2012 onward. Google was thus pursued as the most pragmatic sampling procedure. The search was initially performed using a university computer, based in London (UK). Using Google as a basis for data collection may shape how results are presented because of the location of the search queries, personalized Google profiles, or cookies saved on the computer. In order to replicate the sampling procedure, the same searches were performed using a public computer (without cookies being stored) at the same university. Those procedures were followed in order to obtain "results as close to the product's default results as possible" (Ballatore, 2015, online).

As the phenomenon of this article is relatively new, only articles published from 2016-2019 were selected for inclusion in order to analyze the most recent discussions. Articles from the first four pages of Google (10 results per page) were selected for analysis in order to select the most popular results (Ballatore, 2015). The following sampling procedure was followed: Articles selected for sample inclusion needed to feature a discussion of artificial intuition that was longer than 300 words. Only articles from professional websites and not from personal homepages (private homepages created by individuals) were selected. Articles needed to fall into a broad definition of tech journalism that catered for an audience with an interest in technology and specifically AI (Brennen et al., 2020; Natale \& Ballatore, 2017). Only articles that sought to explain artificial intuition were included. A total of 29 articles were sampled (see annex table for an overview of all sampled articles). A small sample was deliberately chosen in order to conduct a qualitative, detailed thematic textual analysis of the data (Mayring, 2000; Saldaña, 2009). A total of seven items are discussed in this article. Qualitative data analysis was conducted as follows: The content was read and then coded into themes using Nvivo, some of which are outlined further in the following sections (Mayring, 2000; Saldaña, 2009). Themes were constructed as summaries of common points made across articles in order to group articles together. The themes were analyzed 
through a qualitative thematic analysis with a specific focus on how AI technologies were being discussed in relation to human subjectivity. We thus paid particular attention to how AI was described in relation to the human (e.g., through evoking human characteristics, such as the body, brain functioning, cognition, or intuition). Two themes were of a more general nature and dealt with what artificial intuition is and how it relates to human intuition. They were included in this article to introduce readers to an analysis of the topic. A third theme in relation to discussions of practical implementations of artificial intuition in games was selected in order to include journalistic discussions beyond definitions or broader narratives. The tables below provide a summary of the items of this article and their themes and coding rules.

TABLE 1 Overview of Sampled Items

\begin{tabular}{|l|l|l|}
\hline Theme and Summary & Number of Items & Coding Rules \\
\hline $\begin{array}{l}\text { \#1 Defining Artificial Intuition: } \\
\text { Defined in relation to human } \\
\text { intuition }\end{array}$ & In this article: Four & $\begin{array}{l}\text { Article must include general } \\
\text { discussions of artificial intuition } \\
\text { which define it }\end{array}$ \\
\hline $\begin{array}{l}\text { \#2 Intuition and Rationality: } \\
\begin{array}{l}\text { Discussed as making Al more } \\
\text { rational and unbiased }\end{array}\end{array}$ & In this article: Two & $\begin{array}{l}\text { Article must include discussions of } \\
\text { Al as becoming more rational and } \\
\text { unbiased when being intuitive }\end{array}$ \\
\hline $\begin{array}{l}\text { \#3 Practical Implications of Artificial } \\
\text { Intuition in Games: Discussed as } \\
\text { surpassing human intuition }\end{array}$ & In this article: Two & $\begin{array}{l}\text { Article must include discussions } \\
\text { of practical implications of Al for } \\
\text { Al-based digital games }\end{array}$ \\
\hline
\end{tabular}

TABLE 2 Further Information on the Sampled Items

\begin{tabular}{|l|l|c|c|}
\hline Title & Outlet/Author & Length and Theme & URL \\
\hline $\begin{array}{l}\text { Deep Learning, Artificial } \\
\text { Intuition and the Quest } \\
\text { for AGI }\end{array}$ & KDNuggets/Carlos Perez & $\begin{array}{c}1,132 \text { words } \\
\# 1\end{array}$ & Link 1 \\
\hline Artificial Intuition & Medium/Kees Groeneveld & $\begin{array}{c}1,739 \text { words } \\
\# 1\end{array}$ & Link 2 \\
\hline Artificial Intuition & Medium/Nell Watson & $\begin{array}{c}677 \text { words } \\
\# 1\end{array}$ & Link 3 \\
\hline $\begin{array}{l}\text { Did A.I. just make the leap } \\
\text { to being intuitive? }\end{array}$ & Inc/Thomas Koulopoulos & $\begin{array}{c}483 \text { words } \\
\# 1 \text { and \#2 }\end{array}$ & Link 4 \\
\hline $\begin{array}{l}\text { Artificial intuition will } \\
\text { supersede artificial } \\
\text { intelligence, experts say }\end{array}$ & NetworkWorld/Patrick Nelson & $\begin{array}{c}561 \text { words } \\
\# 2\end{array}$ & Link 5 \\
\hline $\begin{array}{l}\text { Building worlds to grow } \\
\text { the artificial mind:The Al } \\
\text { petri-dish }\end{array}$ & Hadean/Rashid Mansoor & $\begin{array}{c}522 \text { words } \\
\# 3\end{array}$ & Link 6 \\
\hline $\begin{array}{l}\text { A computer's newfound } \\
\text { "intuition" beats world } \\
\text { poker champs }\end{array}$ & CNN Health/Michael Nedelman & $\begin{array}{c}1,378 \text { words } \\
\# 3\end{array}$ & Link 7 \\
\hline
\end{tabular}


While a larger sample might have been beneficial, this article focuses on detailed discussion and therefore features a limited amount of data. Future research could devote more scope for further empirical analyses. A more complex sampling procedure could also be followed (e.g., by including tabloid and broadsheet newspapers of specific countries).

\section{Defining Artificial Intuition}

Eight items in the sample discuss artificial intuition in a more general manner. For instance, Groeneveld (2018) makes the argument when writing about Deep Learning and AI that such a type of AI is actually a form of artificial intuition, because we do not fully know how Deep Learning networks come to acquire their knowledge. Frank Pasquale (2015) and others have critically discussed AI through the metaphor of the black box. They argue that, for example, AI-based algorithms deliberately obfuscate their operations from users, competitors, and governments in the interests of competition, surveillance, and profit maximization (see also Burrell, 2016; Cheney-Lippold, 2017; Finn, 2017). The journalistic outlets examined are less critical and instead are more celebratory in tone. This may be because of the wider ways in which technology is often framed by such outlets as something inherently positive and useful for humans, as scholars have pointed out (Bourne, 2019; Brennen et. al., 2020; Goode, 2018; Natale \& Ballatore, 2017). This points to a problem of such narratives in which the appearance of $\mathrm{AI}$ is mistaken for its essence: it is argued that AI can make intuitive decisions which are human-like. "They [neural networks] are able to creatively fill in gaps and make intuitive leaps to make an appropriate response to a given situation" (Watson, 2017, online), writes one commentator, for example. An article on the tech website KDnuggets similarly suggests:

Deep Learning systems exhibit behavior that appears biological despite not being based on biological material. It so happens that humanity has luckily stumbled upon Artificial Intuition in the form of Deep Learning. (Perez, 2017, online)

The equation between humans and AI based on the appearance or particular exhibition of certain processes is simplistic. Intuition is narrowly characterized as something computational:

First off, intuition is just a label we use for a correct decision that's based on incomplete knowledge. We're okay if people are intuitive, in fact we elevate and admire them for it, but we're unsettled by the prospect of a machine making a decision that involves intuition, ambiguity, or less than complete data. But what if our gut is nothing more than a bunch of variables that we're not consciously aware of? (Koulopoulos, 2017, online)

Such a computational view of the human subject is quite different to how human intuition is seen in the humanities and specifically in psychoanalysis. While intuition is generally seen as a positive characteristic of humans, a psychoanalytic view complicates this. For psychoanalysis, intuition is an unconscious process (Jung, 1977). The psychoanalyst 
C. G. Jung (1977) defined intuition as "perception via the unconscious" (p. 306) where one may be perceiving something without conscious awareness. Broadly speaking, one of the goals of psychoanalytic therapy is for individuals to understand better why they acted impulsively, intuitively, and emotionally in particular situations. It understands intuition as being shaped by an individual's biography and their unconscious fantasies and actions. The patient enters into therapy as they suffer because of past or present conflicts, experiences, or relations. They may act intuitively and subsequently understand their intuition better through talking to a psychoanalyst. Unconscious processes can never be rendered completely conscious and some core of the unconscious will always remain (Freud, 1981; Jung, 1977). We evoke the clinical setting at this point because it illustrates the complexity of intuition that AI does not have. In that sense, AI would not act intuitively in a certain situation as a human might because of a past trauma or specific unconscious experience. A psychoanalytic view of intuition and human subjectivity helps to contrast it with the very different understanding of artificial intuition that is put forward in the above narratives. While artificial intuition may appear human-like, it cannot reach the complexity of human intuition as it is unique to each individual. Deploying intuition as a notion or model for AI is therefore problematic.

Based on the definitions of intuition in some of the journalistic outlets, commentators misrecognize intuition as a technicality that can be added to AI in order to make it more flexible, dynamic, and autonomous. As discussed earlier, for cultural theorists, intuition is about an immediate affective and sensorial engagement with the world (Berlant, 2008; Pedwell, 2019; Seigworth, 2006). For commentators on AI, it is portrayed as a technicality that should be built. The intuitiveness of AI is code-based. AI cannot sense intuitively in the same way a human can. Intuition is something that would be programmed into AI. It would be defined through language (code) what and how AI can act intuitively. This shows the limits of true intuition, because for cultural studies scholars the term refers to sensual, affective processes that are in tension with or beyond the discursive. Furthermore, humans cannot be taught to be intuitive; they cannot follow a set of pre-defined rules in order to perfect a sense of intuition. Neither could AI. Psychoanalysis understands intuition as associative. Rather than seeing intuitions as forms of unconscious pattern recognition, as some computer scientists do, intuitions can be regarded as "apprehensions for which one has no conscious reason" (Perkins, 1976, p. 120).

What is at stake here, then, is the deployment of intuition with good intentions by journalists (following technical developments in AI) but its underlying technicality may ultimately harm both humans and AI. Artificial intuition becomes just another form of rationality with little connection to how it is understood in other disciplines. We unpack this point further in the following pages.

\section{Intuition, Unbiased Rationality, and Transparency}

Artificial intuition is in all of the sampled articles regarded as something that can make AI more effective and which would ultimately require less human supervision or control. A journalist writes about the development of a planning algorithm at MIT: "The school recently said it now knows how to include human intuition in a machine algorithm. That's 
a big deal" (Nelson, 2017, online). Such sentences suggest that intuition can be coded into AI. An article on Inc.com similarly states:

AI is actually very well suited to making those sorts of highly intuitive decisions. Since it's not conscious, it has no bias as to what it observes and therefore it's aware of everything that influences a particular decision. (Koulopoulos, 2017, online)

The above author argues that AI is unbiased in its observation of its surroundings. However, AI is far from being unbiased. AI-powered systems have been built by humans, with inherent biases, in the first place and those are often (un)consciously built into the technology (Cheney-Lippold, 2017; Noble, 2018; West et al., 2019). Such narratives also leave out sociocultural aspects of humans and AI; for example, biases of AI systems against certain individuals. Such biases mirror wider social inequalities and stereotypes as they are (un) consciously coded into AI. The question if, for example, a neural network can make a decision based on a hunch is significant, because it can potentially grant even more power to such systems. Researchers have pointed to the ineffectiveness and racialized bias of various AI systems (West et al., 2019). Such ethical questions were largely absent from the items in the sample.

The issue of intuition also relates to wider debates concerning transparency and explainability in AI (Burrell, 2016; Felzmann et al., 2019). AI systems log and store their own decisions and processes, so that humans can scrutinize and troubleshoot them. In that sense, AI is always conscious (aware) of its own processes. However, it is often not possible to explain to lay people how particular decisions have been made by AI, because they are too complex. Intuition would only add to this, because something partly unexplainable through discourse would be needed to be explained by AI (or humans) in order for it to be accountable. This complicates the notion of "retrospective transparency" of AI. Felzmann et al. (2019) define retrospective transparency as a process that "reveals for a specific case how and why a certain decision was reached, describing the data processing step by step" (p. 2). This means that individuals should be able to understand the different factors that led to particular decisions of an AI (both input- and output-related). However, as Felzmann et al. point out, notions of transparency, as they show themselves in practices of informed consent, for example, often assume a rational and fully autonomous individual (Johanssen, 2019). Yet, there are limits to human rationality, as we have also discussed via our psychoanalytic considerations of subjectivity. Individuals may not always be able or willing to understand complex technological processes (Kemper \& Kolkman, 2018). Expectations around transparency also differ according to the specifics of a particular AI (e.g., a social media algorithm or a self-driving car). Users may still trust technology even if they cannot be fully informed about its use (Heald, 2006). However, if the "willingness to be accountable" is seen as "a core indicator of trustworthiness" of AI (Felzmann et al., 2019, p. 10), it remains questionable how such a willingness could be achieved in the case of artificial intuition.

Such questions further complicate ethical considerations of artificial intuition. There is a danger in advocating intuitive AI because intuition is by definition difficult, if not impossible, to explain and account for. If AI acted truly intuitively, this could serve as a justification for being intransparent and opaque, because particular intuitive actions could not 
be explained. It would further the perception of AI as a black box (Pasquale, 2015). Intuition without accountability and transparency would be dangerous. This would also lead to declining trust in AI (Felzmann et al., 2019).

Going beyond the technical aspects of artificial intuition, how the term is (mis)used in tech outlets shows a particular understanding of human subjectivity. AI is not only anthropomorphized and made more human through the advocacy for intuition, at the same time, humans are made more machinic, algorithmic, and technical than they really are in the data we examine. This is done through formulations such as "intuition is just a label we use for a correct decision that's based on incomplete knowledge," our gut being "a bunch of variables" that we are "not consciously aware of" (Koulopoulos, 2017, online). The human subject is regarded in a one-dimensional, functionalist way. Humans and AI function in the same ways and are almost interchangeable. Such equations do not do justice to the complexity of human subjectivity. The human mind is more contradictory and messier than a computer (Turkle, 2005).

Such narratives are similar to the ones of the computer scientists we discussed earlier which suggest that artificial intuition is something that can be coded based on human intuition. Such an argument is too simplistic and while it may speak to the desire of mapping human characteristics onto AI, there is a risk in understanding the human being only in terms that come from computer science. Human subjectivity, in particular, is not only grounded in rationality and language.

\section{Applications of Artificial Intuition: Games}

But what does it really mean for AI to act intuitively? How is intuition understood and framed in such commentaries in relation to specific applications or forms of usage of AI? The last theme we discuss here is the specific application of artificial intuition to digital games. Alphabet's AlphaGo and AlphaGo Zero have recently made headlines for their ability to defeat professional players of the game Go:

AlphaGo Zero often optimises for better board position with virtually utter disregard for piece value. Interestingly, this stems from its ability to teach itself rather than observe human play and thus avoid contamination from human biases. [...] It relies more strongly on heuristics or intuition, needing to look at fewer board positions to arrive at clever strategies. (Mansoor, 2018, online)

Another article discusses AI used for playing poker against humans:

To form DeepStack's so-called intuition, Bowling and his team ran millions of test games against the AI. [...] [P]layers may bluff to hide bad cards, but those are situations DeepStack has already accounted for through math. (Nedelman, 2017, online)

While the need for intuitive AI systems may be comprehensible, the question remains if there is not a contradiction in terms present here. While human intuition is based on experience and the accumulation of enough data to act intuitively in a given situation, how and why the data or experiences were drawn upon when someone acts intuitively is far more 
fuzzy and complex than the above narratives portray. AlphaGo and DeepStack (which are heavily based on neural networks) may have been able to act more intuitively and teach themselves new strategies, but that is hardly the same as human intuition as such. In fact, as Paolo Bory (2019) discusses, the move that led to the defeat of the world champion by the original AlphaGo (the previous version of AlphaGo Zero) was considered as creative, even beautiful by commentators. It was also seen as very un-humanlike. "The day after [the match], DeepMind revealed that AlphaGo decided to play that move for this very reason since the possibility that a human player would play that move was 1:10,000" (Bory, 2019, p. 10). This explanation of an AI "deciding" to perform a particular move, is rooted in logic rather than intuition. A calculation of probabilities led to a particular move that led to the defeat of the human player. The other two quotations about AlphaGo Zero and DeepStack reproduced earlier similarly describe intuition as logical and math-based. Artificial intuition is seen as something that can surpass human intuition in advancing a form of intuition that is unlike those of humans. However close to intuition the moves by AlphaGo Zero or DeepStack may have been, the data that they trained themselves with were still accessible to them. Both systems consciously learned from data.

The narratives discussed in this section point to a different understanding of artificial intuition to the one we have discussed in the previous sections of this article. Whereas many commentators see artificial intuition as something that can be based on human intuition, the discussions of its application in game AIs frame it as something that can actually advance, if not surpass, human intuition as a form of un-humanlikeness. Such narratives suggest a desire for advancing human subjectivity through AI rather than merely mimicking it. Human beings are implicitly seen as deficient or suboptimal in such narratives and it is AI that can advance or assist them. In their work on popular and scientific definitions of social robots, Sarrica et al. (2019) similarly note that "popular online definitions tend to frame social robots as fully autonomous agents, while the scientific literature underscores the fact that they are purpose-built artefacts, entities that execute specific tasks and are only functionally autonomous" (p. 16).

Such narratives are in line with research on media coverage of AI discussed earlier which cover the subject in a future-oriented way that is often somewhat similar to describing science-fiction scenarios, and unlike how scientific discourses would operate (Bourne, 2019; Natale \& Ballatore, 2017). The particular journalistic discourses on AI we have presented also need to be seen in relation to the websites they are published in; tech journalism is a particular genre of journalism that Hanusch (2012) links to lifestyle journalism, a genre he defines as providing information to audiences "often in entertaining ways, about goods and services they can use in their daily lives" (p. 4). Brennen et. al. (2020) note that for tech journalists, "story ideas come from technology 'insiders' in the form of company announcements, blogs, or personal Twitter accounts" (p. 15). There is thus a danger of being influenced by industry PR (see also Bourne, 2019). The narratives we have analyzed in this article often read like exaggerated claims of the AI industry in relation to what artificial intuition could do in the future (Sarrica et al., 2019).

While the desire to advance human subjectivity through AI may be comprehensible, it nonetheless presents ethical challenges in relation to transparency. It also presents practical challenges in relation to the amount of control over AI that humans would retain should they be outsmarted by intuitive systems. It is beyond our ability to assess the technical probability 
of artificial intuition, but how it is portrayed and envisioned in the sampled articles is problematic insofar as important ethical and philosophical notions are not considered.

\section{Conclusion}

It was the goal of this article to analyze how artificial intuition is discussed in tech journalism. We were particularly interested to explore what such narratives reveal about the relationship between humans and AI, as well as what kind of understanding of the human subject is implicitly and explicitly shown in the data. One limitation of this study is its exploratory nature and, therefore, small data set that was examined. Future research should include a larger sample in order to discuss a wider variety of sources. The specific styles, orientations, and target audiences of different outlets that focus on technology journalism should also be examined in more detail. Future research could also include tabloid and broadsheet newspapers of specific countries, for example. Comparisons between tech journalism and more mainstream forms of journalism could then be made.

While we cannot adequately assess the technological feasibility of the phenomenon discussed in this article, we argue that it points to particular desires in how AI technology should progress. In a sense, AI seeks to adopt human characteristics both ontologically and epistemologically. Artificial intuition is an example of making AI appear more efficient, autonomous, human, and flexible than it currently is.

There is a particular understanding, or worldview, of the human subject present in the data we have examined. An understanding that sees the human subject as rational and as being a blueprint, ready to be exploited by AI technology so that humans may be enhanced or, as some accounts argue, ultimately surpassed. Something as complex and difficult to define as intuition is rendered into a mechanized concept for the sake of framing AI in particular ways. We have contrasted such views of the human subject with a psychoanalytic prism which conceptualizes the human as irrational, intuitive, emotional, and as being shaped by trauma and conflict.

The hype around artificial intuition may also have been created by the industry because it not only makes AI appear more human, but also more empathic and likeable (Bory, 2019; McStay, 2018). This might be done deliberately in order to mask transparency, explainability, and accountability of AI. As we have discussed, such a scenario raises crucial ethical questions about the transparency of AI.

Intuition, then, is not the best term to make very valid arguments about the current rigidness or limitations of AI. AI needs to become more flexible-rather than intuitive-and this should happen in an ethical and transparent manner.

\section{Author Biographies}

Jacob Johanssen $(\mathrm{PhD})$ is Senior Lecturer in Communications, St. Mary's University (London, UK). He is the author of Psychoanalysis and Digital Culture: Audiences, Social Media, and Big Data (Routledge, 2019). His research interests include psychoanalysis and digital media, emerging technologies and artificial intelligence, psychosocial studies, and critical theory. 
Xin Wang $(\mathrm{PhD})$ is Tenure-Track Professor of Media and Cultural Studies, College of Arts and Media, Tongji University (Shanghai, People's Republic of China). Her research interests are Media and Cultural Studies, intercultural communication, critical theory and media philosophy.

\section{References}

Ballatore, A. (2015). Google chemtrails: A methodology to analyze topic representation in search engine results. First Monday, 20(7).

Berlant, L. (2008). Intuitionists: History and the affective event. American Literary History, 20(4), 845-860. https://doi.org/10.1093/alh/ajn039

Board, R. (1958). Intuition in the methodology of psychoanalysis. Psychiatry, 21(3), $233-$ 239. https://doi.org/10.1080/00332747.1958.11023132

Bory, P. (2019). Deep new: The shifting narratives of artificial intelligence from Deep Blue to AlphaGo. Convergence. https://doi.org/10.1177/1354856519829679

Bourne, C. (2019). AI cheerleaders: Public relations, neoliberalism and artificial intelligence. Public Relations Inquiry, 8(2), 109-125. https://doi.org/10.1177/2046147X19835250

Brennen, S., Howard, P. N., \& Kleis Nielsen, R. (2018). An industry-led debate: How UK media cover artificial intelligence. Report. https://reutersinstitute.politics.ox.ac.uk/sites/ default/files/2018-12/Brennen_UK_Media_Coverage_of_AI_FINAL.pdf

Brennen, S., Howard, P. N., \& Kleis Nielsen, R. (2020). Balancing product reviews, traffic targets, and industry criticism: UK technology journalism in practice. Journalism Practice. https://doi.org/10.1080/17512786.2020.1783567

Burrell, J. (2016). How the machine 'thinks': Understanding opacity in machine learning algorithms. Big Data \& Society, 3(1), 1-12. https://doi.org/10.1177/2053951715622512

Caudill, M., \& Butler, C. (1990). Naturally intelligent systems. MIT Press.

Cave, S., Craig, C., Dihal, K. S., Dillon, S., Montgomery, J., Singler, B., \& Taylor, L. (2018). Portrayals and perceptions of AI and why they matter. London: The Royal Society. https://www.repository.cam.ac.uk/handle/1810/287193

Cheney-Lippold, J. (2017). We are data: Algorithms and the making of our digital selves. NYU Press.

Chudnoff, E. (2013). Intuition. Oxford University Press.

Crowder, J., \& Friess, S. (2013). Artificial psychology: The psychology of AI. Proceedings of the 3rd Annual International Multi-Conference on Informatics and Cybernetics. Orlando, FL.

Diaz-Hernandez, O., \& Gonzalez-Villela, V. J. (2015). Analysis of human intuition towards artificial intuition synthesis for robotics. Mechatronics and Applications: An International Journal (MECHATROJ), 1(1), 23-39. https://doi.org/10.2139/ssrn.3427647

Dundas, J., \& Chik, D. (2011): Ibsead: - A self-evolving self-obsessed learning algorithm for machine learning. International Journal of Computer Science \& Emerging Technologies, 1(4), 74-79. https://arxiv.org/ftp/arxiv/papers/1106/1106.6186.pdf

Felzmann, H., Fosch Villaronga, E., Lutz, C., \& Tamo-Larrieux, A. (2019). Transparency you can trust: Transparency requirements for artificial intelligence between legal norms and contextual concerns. Big Data \& Society, 6(1), 1-14. https://doi. org/10.1177/2053951719860542 
Finn, E. (2017). What algorithms want: Imagination in the age of computing. MIT Press.

Frantz, R. (2003). Herbert Simon. Artificial intelligence as a framework for understanding intuition. Journal of Economic Psychology, 24, 265-277. https://doi.org/10.1016/ S0167-4870(02)00207-6

Freud, S. (1981). Five lectures on psycho-analysis. The Standard Edition of the Complete Psychological Works of Sigmund Freud. Volume XI. Five Lectures on Psycho-Analysis, Leonardo Da Vinci and Other Works. The Hogarth Press and the Institute of Psycho-Analysis.

Goode, L. (2018). Life, but not as we know it: AI and the popular imagination. Culture Unbound: Journal of Current Cultural Research, 10(2), 185-207. http://www.culture unbound.ep.liu.se/v10/a14/cu18v10a14.pdf

Groeneveld, K. (2018). Artificial Intuition. Medium. https://medium.com/@casegreenfield/ artificial-intuition-784963f989be

Hanusch, F. (2012). Broadening the focus: The case for lifestyle journalism as a field of scholarly inquiry. Journalism Practice, 6(1), 2-11. https://doi.org/10.1080/17512786.20 11.622895

Heald, D. A. (2006). Transparency as an instrumental value. In C. Hood \& D. Heald (Eds.), Transparency: The key to better governance? Proceedings of the British Academy (vol. 135, pp. 59-73). Oxford University Press.

Johanssen, J. (2019). Psychoanalysis and digital culture: Audiences, social media, and big data. Routledge.

Johnny, O., Trovati, M., \& Ray, J. (2019). Toward a computational model of artificial intuition and decision making. In L. Barolli, H. Nishino, H. Miwa (Eds.), Advances in Intelligent Networking and Collaborative Systems. The 11th International Conference on Intelligent Networking and Collaborative Systems (INCoS-2019). Springer Nature, 463-472.

Jung, C. G. (1977). C.G. Jung speaking: Interviews and encounters. Princeton University Press.

Kemper, J., \& Kolkman, D. (2018). Transparent to whom? No algorithmic accountability without a critical audience. Information, Communication \& Society, 22(14), 2081-2096. https://doi.org/10.1080/1369118X.2018.1477967

Koulopoulos, T. (2017). Did A.I. just make the leap to being intuitive? Inc.com. https://www. inc.com/thomas-koulopoulos/did-ai-just-make-leap-to-being-intuitive.html

Mansoor, R. (2018). Building worlds to grow the artificial mind: The AI petri-dish. Hadean.

Mayring, P. (2000). Qualitative content analysis. Forum Qualitative Social Research, 1(2), http://www.qualitative-research.net/index.php/fqs/article/view/1089/2385

McStay, A. (2018). Emotional AI: The rise of empathic media. Sage.

Natale, S., \& Ballatore, A. (2017). Imagining the thinking machine: Technological myths and the rise of artificial intelligence. Convergence, 26(1), 3-18. https://doi. org/10.1177/1354856517715164

Nedelman, M. (2017). A computer's newfound 'intuition' beats world poker champs. CNN Health. https://edition.cnn.com/2017/03/02/health/artificial-intelligence-poker-intuitionstudy/index.html

Nelson, P. (2017). Artificial intuition will supersede artificial intelligence, experts say. Network World. https://www.networkworld.com/article/3168320/artificial-intuition-will-supersedeartificial-intelligence-experts-say.html 
Noble, S. U. (2018). Algorithms of oppression: How search engines reinforce racism. New York University Press.

Pasquale, F. (2015). The black box society. The secret algorithms that control money and information. Harvard University Press.

Pedwell, C. (2019). Digital tendencies: Intuition, algorithmic thought and new social movements. Culture, Theory and Critique, 60(2), 123-138. https://doi.org/10.1080/14735784. 2019.1579658

Perez, C. (2017). Deep learning, artificial intuition and the quest for AGI. KDnuggets. https://www.kdnuggets.com/2017/02/deep-learning-artificial-intelligence-quest-agi.html

Perkins, D. N. (1976). The limits of intuition. Leonardo, 10(2), 119-125.

Rosenblatt, A. D., \& Thickstun, J. T. (1994). Intuition and consciousness. The Psychoanalytic Quarterly, 63(4), 696-714. https://doi.org/10.1080/21674086.1994.11927433

Russell, S., \& Norvig, P. (2010). Artificial intelligence: A modern approach (3rd ed.). Pearson. Saldaña, J. (2009). The coding manual for qualitative researchers. Sage.

Sarrica, M., Brondi, S., \& Fortunati, L. (2019). How many facets does a "social robot" have? A review of scientific and popular definitions online. Information Technology \& People, 33(1), 1-21. https://doi.org/10.1108/ITP-04-2018-0203

Seigworth, G. J. (2006). Cultural studies and Gilles Deleuze. In G. Hall and C. Birchall (Eds.), New Cultural Studies: Adventures in Theory (pp. 107-126). Edinburgh University Press.

Srdanov, A., Kovačević, N. R., Vasić, S., \& Milovanović, D. (2016). Emulation of artificial intuition using random choice and logic. In 2016 13th Symposium on Neural Networks and Applications (NEUREL) (pp. 1-6). IEEE. https://doi.org/10.1109/NEUREL.2016.7800114

Szalita-Pemow, A. B. (1955). The "intuitive process" and its relation to work with schizophrenics. Journal of the American Psychoanalysis Association, 3(1), 7-18. https://doi. org/10.1177/000306515500300102

Tao, W., \& He, P. (2009). Intuitive learning and artificial intuition networks. 2009 Second International Conference on Education Technology and Training (pp. 297-300). IEEE. https://ieeexplore.ieee.org/abstract/document/5381516

Turkle, S. (2005). The second self: Computers and the human spirit (Twentieth anniversary ed.). MIT Press.

Turner, J. (2019). Robot rules: Regulating artificial intelligence. Palgrave Macmillan.

Watson, N. (2017). Artificial intuition. The limitations (and ridiculous power) of ANN creativity. Medium. https://medium.com/@nellwatson/artificial-intuition-928c3a43ec2a

West, S. M., Whittaker, M., and Crawford, K. (2019). Discriminating systems: Gender, race and power in AI. AI Now Institute. https://ainowinstitute.org/discriminatingsystems. html

Wouters, P., \& Gerbec, D. (2006). Interactive internet? Studying mediated interaction with publicly available search engines. Journal of Computer-Mediated Communication, 8(4). https://doi.org/10.1111/j.1083-6101.2003.tb00221.x 
Annex Table: Complete List of Sampled Articles

\begin{tabular}{|c|c|c|c|}
\hline Title & Author/Outlet & Theme(s) & URL \\
\hline Artificial Intuition & $\begin{array}{l}\text { Kees Groeneveld/ } \\
\text { Medium }\end{array}$ & $\begin{array}{l}\text { Defining Artificial } \\
\text { Intuition }\end{array}$ & Link 1 \\
\hline Artificial Intuition & $\begin{array}{l}\text { Medium/Nell } \\
\text { Watson }\end{array}$ & $\begin{array}{l}\text { Defining Artificial } \\
\text { Intuition }\end{array}$ & Link 2 \\
\hline $\begin{array}{l}\text { Next-Generation Al Could Develop } \\
\text { Its Own "Human Intuition" }\end{array}$ & $\begin{array}{l}\text { Dyani Sabin/ } \\
\text { Inverse }\end{array}$ & $\begin{array}{l}\text { Defining Artificial } \\
\text { Intuition; Future; Al } \\
\text { and Humans }\end{array}$ & Link 3 \\
\hline $\begin{array}{l}\text { Deep Learning, Artificial Intuition } \\
\text { and the Quest for AGI }\end{array}$ & $\begin{array}{l}\text { Carlos E. Perez/ } \\
\text { KDnuggets }\end{array}$ & $\begin{array}{l}\text { Defining Artificial } \\
\text { Intuition }\end{array}$ & Link 4 \\
\hline $\begin{array}{l}\text { Tech Experts Predict Artificial } \\
\text { Intuition Will Be the New Trend In } \\
\text { Artificial Intelligence }\end{array}$ & $\begin{array}{l}\text { Victor Thomson/ } \\
\text { itechpost }\end{array}$ & $\begin{array}{l}\text { Defining Artificial } \\
\text { Intuition; Future }\end{array}$ & Link 5 \\
\hline $\begin{array}{l}\text { How Artificial Intelligence Will Take } \\
\text { Over the Supermarket Produce } \\
\text { Aisles }\end{array}$ & $\begin{array}{l}\text { Joseph Byrum/ } \\
\text { TechCrunch }\end{array}$ & $\begin{array}{l}\text { Defining Artificial } \\
\text { Intuition; Business }\end{array}$ & Link 6 \\
\hline $\begin{array}{l}\text { Artificial Intuition Wants to Guide } \\
\text { Business Decisions. Can It Improve } \\
\text { on "Going With Your Gut"? }\end{array}$ & $\begin{array}{l}\text { Alyssa Newcomb/ } \\
\text { Fortune }\end{array}$ & Business & Link 7 \\
\hline $\begin{array}{l}\text { Meet Velas: When Artificial Intuition } \\
\text { Boosts Blockchain Capabilities }\end{array}$ & $\begin{array}{l}\text { Alex Dovbnya/ } \\
\text { UToday }\end{array}$ & Business; Technology & Link 8 \\
\hline $\begin{array}{l}\text { Node Launches Artificial Intuition } \\
\text { Platform to Predict Business } \\
\text { Outcomes }\end{array}$ & $\begin{array}{l}\text { No author/BW } \\
\text { BusinessWorld }\end{array}$ & Business & Link 9 \\
\hline $\begin{array}{l}\text { Blockchain 3.0-Taking DLT to the } \\
\text { Next Level With Al-Integration }\end{array}$ & $\begin{array}{l}\text { Hackermoon/David } \\
\text { Cullinan }\end{array}$ & Business; Technology & Link 10 \\
\hline $\begin{array}{l}\text { Next-Gen Software Aims to Lift } \\
\text { Personal Computer Into Aware State }\end{array}$ & $\begin{array}{l}\text { Nancy Cohen/ } \\
\text { TechXplore }\end{array}$ & Technology & Link 11 \\
\hline How Do You Code Intuition Into Al? & No author/Loop54 & $\begin{array}{l}\text { Technology; Intuition } \\
\text { and Rationality }\end{array}$ & Link 12 \\
\hline $\begin{array}{l}\text { Helping Machines Understand Laws } \\
\text { of Physics for Intuition on How } \\
\text { Objects Should Behave }\end{array}$ & $\begin{array}{l}\text { Rob Matheson/ } \\
\text { SciTechDaily }\end{array}$ & Technology & Link 13 \\
\hline $\begin{array}{l}\text { Artificial Intuition and } \\
\text { Reinforcement Learning, the Next } \\
\text { Steps in Machine Learning }\end{array}$ & $\begin{array}{l}\text { Vince Tabora/ } \\
\text { BecomingHumanAl }\end{array}$ & Technology; Future & Link 14 \\
\hline $\begin{array}{l}\text { From Intellect to Intuition: What } \\
\text { Happens When We Humanise AI } \\
\text { Systems? }\end{array}$ & $\begin{array}{l}\text { Nick Ismail/ } \\
\text { Information Age }\end{array}$ & $\begin{array}{l}\text { Defining Artificial } \\
\text { Intuition; Future }\end{array}$ & Link 15 \\
\hline $\begin{array}{l}\text { Artificial Intelligence Goes Deep to } \\
\text { Beat Humans at Poker }\end{array}$ & $\begin{array}{l}\text { Tonya Riley/ Science } \\
\text { Mag }\end{array}$ & Games & Link 16 \\
\hline
\end{tabular}




\begin{tabular}{|c|c|c|c|}
\hline Title & Author/Outlet & Theme(s) & URL \\
\hline $\begin{array}{l}\text { A Computer's Newfound "Intuition" } \\
\text { Beats World Poker Champs }\end{array}$ & $\begin{array}{l}\text { Michael Nedelman/ } \\
\text { CNN Health }\end{array}$ & Games & Link 17 \\
\hline $\begin{array}{l}\text { Building Worlds to Grow the } \\
\text { Artificial Mind: The AI Petri-Dish }\end{array}$ & $\begin{array}{l}\text { Hadean/Rashid } \\
\text { Mansoor }\end{array}$ & Games & Link 18 \\
\hline $\begin{array}{l}\text { Scientists Teach Computers Fear- } \\
\text { To Make Them Better Drivers }\end{array}$ & $\begin{array}{l}\text { Patrick Watson/ } \\
\text { Science Mag }\end{array}$ & Feelings & Link 19 \\
\hline Al: Cars With "Gut Feelings" & Arnold Schlegel/ ZF & Feelings & Link 20 \\
\hline Automating the Future of Design & Jim Bull/Forbes & Feelings; Business & Link 21 \\
\hline $\begin{array}{l}\text { MIT Incorporates Human Intuition } \\
\text { in Artificial Intelligence to Help } \\
\text { Computers Plan Better }\end{array}$ & $\begin{array}{l}\text { No author/ Firstpost } \\
\text { Tech2 }\end{array}$ & Al and Humans & Link 22 \\
\hline $\begin{array}{l}\text { Can Artificial Intelligence Replace } \\
\text { Human Intuition? }\end{array}$ & $\begin{array}{l}\text { Upasana } \\
\text { Bhattacharjee/ } \\
\text { Qrius }\end{array}$ & Al and Humans & Link 23 \\
\hline $\begin{array}{l}\text { Al and Human Intuition Go Hand in } \\
\text { Hand }\end{array}$ & $\begin{array}{l}\text { Yue Wu/Venture } \\
\text { Beat }\end{array}$ & Al and Humans & Link 24 \\
\hline $\begin{array}{l}\text { Modular Deep Learning Could } \\
\text { Be the Penultimate Step to } \\
\text { Consciousness }\end{array}$ & $\begin{array}{l}\text { Carlos E. Perez/ } \\
\text { Medium }\end{array}$ & Al and Humans & Link 25 \\
\hline $\begin{array}{l}\text { Are You Intuitive? Challenge My } \\
\text { Machine! }\end{array}$ & $\begin{array}{l}\text { John David Martin/ } \\
\text { Hackermoon }\end{array}$ & $\begin{array}{l}\text { Al and Humans; } \\
\text { Technology }\end{array}$ & Link 26 \\
\hline $\begin{array}{l}\text { Artificial Intuition Will Supersede } \\
\text { Artificial Intelligence, Experts Say }\end{array}$ & $\begin{array}{l}\text { Patrick Watson/ } \\
\text { NetworkWorld }\end{array}$ & $\begin{array}{l}\text { Al and Humans; } \\
\text { Intuition and } \\
\text { Rationality; Future }\end{array}$ & Link 27 \\
\hline $\begin{array}{l}10 \text { Experts on the Future of Artificial } \\
\text { Intelligence }\end{array}$ & $\begin{array}{l}\text { Anirudh V. K./ } \\
\text { Toolbox }\end{array}$ & Future & Link 28 \\
\hline $\begin{array}{l}\text { Did A.I. Just Make the Leap to Being } \\
\text { Intuitive? }\end{array}$ & $\begin{array}{l}\text { Inc/Thomas } \\
\text { Koulopoulos }\end{array}$ & $\begin{array}{l}\text { Defining Artificial } \\
\text { Intuition; Intuition } \\
\text { and Rationality; } \\
\text { Future; Technology }\end{array}$ & Link 29 \\
\hline
\end{tabular}

